Research Article

\title{
An improved lower bound for the degree Kirchhoff index of bipartite graphs
}

\author{
Ş. Burcu Bozkurt Altındağ ${ }^{1, *}$, Igor Milovanović ${ }^{2}$ Emina Milovanović ${ }^{2}$, Marjan Matejić ${ }^{2}$
}

${ }^{1}$ Yenikent Kardelen Konutlarl, Selçuklu, 42070 Konya,Turkey

${ }^{2}$ Faculty of Electronic Engineering, University of Niš, Niš, Serbia

(Received: 8 November 2021. Received in revised form: 15 January 2022. Accepted: 15 January 2022. Published online: 18 January 2022.)

(C) 2022 the authors. This is an open access article under the CC BY (International 4.0) license (www.creativecommons.org/licenses/by/4.0/).

\begin{abstract}
For a connected graph $G$ with $n$ vertices and $m$ edges, the degree Kirchhoff index of $G$ is defined as $K f^{*}(G)=2 m \sum_{i=1}^{n-1}\left(\gamma_{i}\right)^{-1}$, where $\gamma_{1} \geq \gamma_{2} \geq \cdots \geq \gamma_{n-1}>\gamma_{n}=0$ are the normalized Laplacian eigenvalues of $G$. In this paper, a lower bound on the degree Kirchhoff index of bipartite graphs is established. Also, it is proved that the obtained bound is stronger than a lower bound derived by Zhou and Trinajstić in [J. Math. Chem. 46 (2009) 283-289].
\end{abstract}

Keywords: topological indices; degree Kirchhoff index.

2020 Mathematics Subject Classification: 05C12, 05C50.

\section{Introduction}

Let $G=(V(G), E(G))$ be a simple connected graph with $n$ vertices and $m$ edges, where $V(G)=\left\{v_{1}, v_{2}, \ldots, v_{n}\right\}$. The degree of a vertex $v_{i} \in V(G)$ is denoted by $d_{i}$, where $i=1,2, \ldots, n$. If $v_{i}$ and $v_{j}$ are two adjacent vertices of $G$, then it is written as $i \sim j$.

Denote by $A(G)$ and $D(G)=\operatorname{diag}\left(d_{1}, d_{2}, \ldots, d_{n}\right)$ the adjacency and the diagonal degree matrix of $G$, respectively. The Laplacian matrix of $G$ is defined as $L(G)=D(G)-A(G)$ (see [16]). Since $G$ is assumed to be a connected graph, the matrix $D(G)^{-1 / 2}$ exists. The normalized Laplacian matrix of $G$ is the matrix defined [8] by

$$
\mathcal{L}(G)=D(G)^{-1 / 2} L(G) D(G)^{-1 / 2} .
$$

The eigenvalues $\gamma_{1} \geq \gamma_{2} \geq \cdots \geq \gamma_{n-1}>\gamma_{n}=0$ of $\mathcal{L}(G)$ represent the normalized Laplacian eigenvalues of $G$. Details on the spectra of $\mathcal{L}(G)$ can be found in [8].

Chen and Zhang [7] introduced the degree Kirchhoff index of a connected graph $G$ as

$$
K f^{*}(G)=\sum_{i<j} d_{i} d_{j} r_{i j},
$$

where $r_{i j}$ is the effective resistance distance between the vertices $v_{i}$ and $v_{j}$ of $G$. In [7], it was also demonstrated that the degree Kirchhoff index can be expressed in terms of normalized Laplacian eigenvalues as follows:

$$
K f^{*}(G)=2 m \sum_{i=1}^{n-1} \frac{1}{\gamma_{i}} .
$$

Both of the definitions of the graph invariant $K f^{*}(G)$ given by (1) and (2) are much studied in the chemical and mathematical literature. For survey and details, see [1,2,4,5,10-12,14,15, 17, 18, 20,21].

In this paper, we present a lower bound on the degree Kirchhoff index of bipartite graphs. In addition, we show that our lower bound improves the lower bound obtained by Zhou and Trinajstić [21].

\section{Lemmas}

In this section, we recall a few well-known properties of the normalized Laplacian eigenvalues of graphs.

\footnotetext{
*Corresponding author (srf_burcu_bozkurt@hotmail.com).
} 
Lemma 2.1. [8] Let $G$ be a connected graph with $n \geq 2$ vertices. Then, the following properties regarding the normalized Laplacian eigenvalues are valid:

1. $\sum_{i=1}^{n} \gamma_{i}=n$.

2. $\gamma_{1} \leq 2$ with equality if and only if $G$ is a bipartite graph.

3. For each $1 \leq i \leq n, \gamma_{i} \in[0,2], \gamma_{n}=0$ and $\gamma_{n-1} \neq 0$.

Lemma 2.2. [9] Let $G$ be a connected graph with $n$ vertices and $m$ edges. Then,

$$
\prod_{i=1}^{n-1} \gamma_{i}=\frac{2 m t(G)}{\prod_{i=1}^{n} d_{i}}
$$

where $t(G)$ is the total number of spanning trees of $G$.

Lemma 2.3. [13] Let $G$ be a connected graph of order $n$. Then, $\gamma_{2} \geq 1$ with equality if and only if $G$ is a complete bipartite graph.

\section{A lower bound for the degree Kirchhoff index of bipartite graphs}

We now give an improved lower bound on the degree Kirchhoff index of bipartite graphs.

Theorem 3.1. Let $G$ be a connected bipartite graph with $n \geq 2$ vertices, $m$ edges and $t(G)$ spanning trees. Then, for any real $\alpha, \gamma_{2} \geq \alpha \geq 1$

$$
K f^{*}(G) \geq 2 m\left(\frac{1}{2}+\frac{1}{\alpha}+n-3-\ln \left(\frac{m t(G)}{\prod_{i=1}^{n} d_{i}}\right)+\ln \alpha\right) .
$$

Equality in (3) holds if and only if $\alpha=1$ and $G \cong K_{p, q}(p+q=n)$.

Proof. For $x>0$, the following inequality can be found in the monograph [19]

$$
x \leq 1+x \ln x,
$$

where the equality holds if and only if $x=1$. For $x>0$, the above inequality can be considered as

$$
\frac{1}{x} \geq 1-\ln x
$$

with equality if and only if $x=1$. By Lemma $2.1, \gamma_{1}=2$ and $\gamma_{i}>0, i=1,2, \ldots, n-1$, since $G$ is a connected bipartite graph. Then, using these results and Lemma 2.2, we have

$$
\begin{aligned}
\sum_{i=1}^{n-1} \frac{1}{\gamma_{i}} & =\frac{1}{\gamma_{1}}+\frac{1}{\gamma_{2}}+\sum_{i=3}^{n-1} \frac{1}{\gamma_{i}} \\
& =\frac{1}{2}+\frac{1}{\gamma_{2}}+\sum_{i=3}^{n-1} \frac{1}{\gamma_{i}} \\
& \geq \frac{1}{2}+\frac{1}{\gamma_{2}}+\sum_{i=3}^{n-1}\left(1-\ln \gamma_{i}\right) \\
& =\frac{1}{2}+\frac{1}{\gamma_{2}}+n-3-\ln \prod_{i=3}^{n-1} \gamma_{i} \\
& =\frac{1}{2}+\frac{1}{\gamma_{2}}+n-3-\ln \left(\frac{m t(G)}{\prod_{i=1}^{n} d_{i}}\right)+\ln \gamma_{2} .
\end{aligned}
$$

Now, consider the function $f(x)=\frac{1}{x}+\ln x$. It can be easily seen that this function is increasing in the interval $1 \leq x \leq 2$. Then for any real $\alpha, \gamma_{2} \geq \alpha \geq 1$, we have that

$$
f\left(\gamma_{2}\right) \geq f(\alpha)=\frac{1}{\alpha}+\ln \alpha .
$$

Bearing this fact in mind and using (2) and (4), we obtain that

$$
K f^{*}(G) \geq 2 m\left(\frac{1}{2}+\frac{1}{\alpha}+n-3-\ln \left(\frac{m t(G)}{\prod_{i=1}^{n} d_{i}}\right)+\ln \alpha\right)
$$


which is the required inequality (3). Now, assume that the equality holds in (3). Then

$$
\gamma_{2}=\alpha \text { and } \gamma_{3}=\cdots=\gamma_{n-1}=1 .
$$

Since $G$ is bipartite, by Lemma $2.1, \sum_{i=2}^{n-1} \gamma_{i}=n-2$. Considering this with the above conditions, we get that $\gamma_{2}=\alpha=1$, which implies that $G \cong K_{p, q}$.

Conversely, it is not difficult to show that the equality holds in (3) for the complete bipartite graph $K_{p, q}$. Hence, the proof is completed.

By Theorem 3.1 and Lemma 2.3, we have the following corollary.

Corollary 3.1. Let $G$ be a connected bipartite graph with $n \geq 2$ vertices, $m$ edges and $t(G)$ spanning trees. Then,

$$
K f^{*}(G) \geq m(2 n-3)-2 m \ln \left(\frac{m t(G)}{\prod_{i=1}^{n} d_{i}}\right) .
$$

Equality in (5) holds if and only if $G \cong K_{p, q}(p+q=n)$.

Remark 3.1. For a connected bipartite graph $G$ with $n \geq 2$ vertices and $m$ edges, Zhou and Trinajstić [21] obtained that

$$
K f^{*}(G) \geq m(2 n-3)
$$

with equality if and only if $G$ is a complete bipartite graph. Furthermore, for connected bipartite graphs, the following inequality can be obtained from Theorem 3 of [3]:

$$
0<\frac{m t(G)}{\prod_{i=1}^{n} d_{i}} \leq 1
$$

From the above and (5), we conclude that

$$
\begin{aligned}
K f^{*}(G) & \geq m(2 n-3)-2 m \ln \left(\frac{m t(G)}{\prod_{i=1}^{n} d_{i}}\right) \\
& \geq m(2 n-3) .
\end{aligned}
$$

This implies that the lower bound (5) improves the lower bound (6).

Recall that the general Randić index of a graph $G$ is one of the graph topological indices defined by $R_{-1}(G)=\sum_{i \sim j} \frac{1}{d_{i} d_{j}}$ (see [6]). The following lower bound was found in Theorem 3.2 of [5]

$$
\gamma_{2} \geq 1+\sqrt{\frac{2\left(R_{-1}(G)-1\right)}{n-2}} .
$$

Remark 3.2. Notice that the lower bound (5) can be improved by taking $\alpha=1+\sqrt{\frac{2\left(R_{-1}(G)-1\right)}{n-2}}$ in Theorem 3.1.

\section{Acknowledgment}

The authors are grateful to two anonymous referees for their valuable comments and suggestions.

\section{References}

[1] M. Bianchi, A. Cornaro, J. L. Palacios, J. M. Renom, A. Torriero, Revisiting bounds for the multiplicative degree-Kirchhoff index, MATCH Commun. Math. Comput. Chem. 75 (2016) 227-231.

[2] M. Bianchi, A. Cornaro, J. L. Palacios, A. Torriero, Bounding the sum of powers of normalized Laplacian eigenvalues of graph through majorization method, MATCH Commun. Math. Comput. Chem. 70 (2013) 707-716.

[3] Ş. B. Bozkurt, Upper bounds for the number of spanning trees of graphs, J. Inequal. Appl. 269 (2012) 1-7.

[4] Ş. B. Bozkurt, D. Bozkurt, On the sum of powers of normalized Laplacian eigenvalues of graphs, MATCH Commun. Math. Comput. Chem. 68 (2012) 917-930.

[5] S. B. Bozkurt Altındağ, I. Milovanović, M. Matejić, E. Milovanović, On the degree Kirchhoff index of bipartite graphs, Sci. Publ. State Univ. Novi Pazar, Ser. A: Appl. Math. Inform. Mech. 13 (2021) 1-8.

[6] M. Cavers, S. Fallat, S. Kirkland, On the normalized Laplacian energy and general Randić index $R_{-1}$ of graphs, Linear Algebra Appl. 433 (2010) $172-190$

[7] H. Chen, F. Zhang, Resistance distance and the normalized Laplacian spectrum, Discrete Appl. Math. 155 (2007) 654-661.

[8] F. R. K. Chung, Spectral Graph Theory, Amer. Math. Soc., Providence, 1997.

[9] D. Cvetković, M. Doob, H. Sachs, Spectra of graphs, Academic press, New York, 1980.

[10] K. C. Das, Y. Yang, K. Xu, Nordhaus-Gaddum-type results for resistance distance-based graph invariants, Discuss. Math. Graph. Theory 36 (2016) 695-707.

[11] L. Feng, I. Gutman, G. Yu, Degree Kirchhoff index of unicyclic graphs, MATCH Commun. Math. Comput. Chem. 69 (2013) $629-648$.

[12] M. Hakimi-Nezhaad, A. R. Ashrafi, I. Gutman, Note on degree Kirchhoff index of graphs, Trans. Combin. 2 (2013) 43-52. 
[13] J. Li, J. M. Guo, W. C. Shiu, Bounds on normalized Laplacian eigenvalues of graphs, J. Inequal. Appl. 316 (2014) 1-8.

[14] J. Li, J. M. Guo, W. C. Shiu, Ş. B. Bozkurt Altındağ, D. Bozkurt, Bounding the sum of powers of normalized Laplacian eigenvalues of a graph, Appl. Math. Comput. 324 (2018) 82-92.

[15] M. Matejić, I. Milovanović, E. Milovanović, Remarks on the degree Kirchhoff index, Kragujevac J. Math. 43 (2019) $15-21$.

[16] R. Merris, Laplacian matrices of graphs: a survey, Linear Algebra Appl. $197 \& 198$ (1994) 143-176.

[17] I. Milovanović, I. Gutman, E. Milovanović, On Kirchhoff and degree Kirchhoff indices, Filomat 29 (2015) $1869-1877$.

[18] I. Milovanović, E. Milovanović, Bounds of Kirchhoff and degree Kirchhoff indices, In: I. Gutman, B. Furtula, K. C. Das, E. Milovanović, I. Milovanović (Eds.), Bounds in Chemical Graph Theory - Mainstreams, Univ. Kragujevac, Kragujevac, 2017, pp. 93-119.

[19] D. S. Mitrinović, Elementary Inequalities, P. Noordhoff, Groningen, 1964.

[20] J. Palacios, J. M. Renom, Another look at the degree Kirchhoff index, Int. J. Quantum Chem. 111 (2011) 3453-3455.

[21] B. Zhou, N. Trinajstić, On resistance-distance and Kirchhoff index, J. Math. Chem. 46 (2009) 283-289. 